NASA Contractor Report 180859

\title{
Performance and Operating Envelope of Imaging and Scattering Particle Sizing Instruments
}

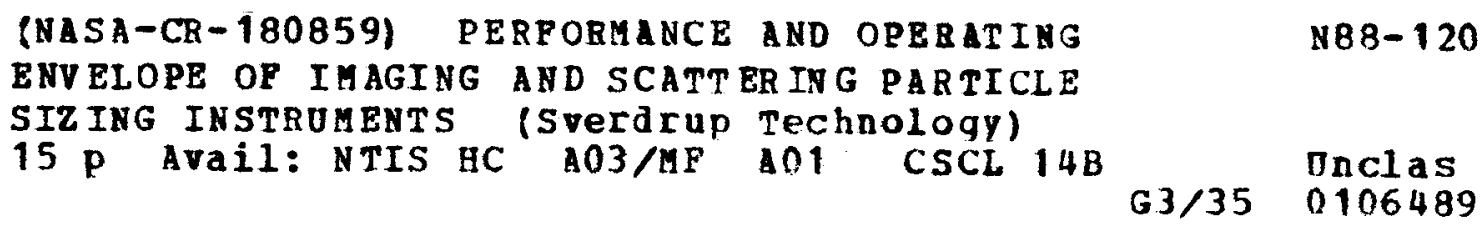

Edward A. Hovenac

Sverdrup Technology, Inc.

Lewis Research Center

Cleveland, Ohio

November 1987

Prepared for

Lewis Research Center

Under Contract NAS3-24105

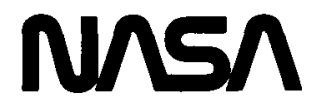

National Aeronautics and

Space Administration 
Performance and operating Envelope of
Imaging and Scattering Particle Sizing Instruments

Edward A. Hovenac

Sverdrup Technology, Inc.

Lewis Research Center

Cleveland, Ohio 44135 USA

\begin{abstract}
scattering and imaging type particle sizing instruments are analyzed in terms of their ability to make accurate determinations of particle size distributions, number density, and total mass. Sources of counting and sizing errors are explained. The paper describes ways of identifying these errors and how these errors can effect the measurement.
\end{abstract}

\title{
Introduction
}

Scattering and imaging are two of the oldest and most widely used techniques for measuring particle size distributions. In this section basic operating principles of these instruments will be discussed, followed by an explanation of how counting and sizing errors effect various measurement quantities, and finally a definition of "operating envelope" will be considered. The second and third sections give details of how measurement conditions and optical effects can shape the operating envelope.

scattering and Imaging Type Instruments

The particle sizing instruments discussed in this paper are optical nonintrusive single particle counters. In several cases throughout the paper, specific instrument examples are used. These examples usually refer to the forward scattering Spectrometer Probe (FSSP) and the optical Array Probe (OAP) manufactured by Particle Measuring systems of Boulder, colorado. These instruments are used as examples because of the author's familiarity with them. Most of the discussion, however is intended for generic scattering and imaging instruments. The particle size range of these instruments is nominally from 0.5 micrometers to several hundred micrometers.

Scattering type particle sizing instruments such as the FSSP measure the diameter of particles by analyzing the intensity of scattered light collected over a solid angle. A laser is used as a light source and is focused in the measurement region. 
Particles are measured one at a time as they cross the focused beam (also called the probe volume or sample volume). As a particle enters the probe volume it begins to scatter light. When it reaches the most intense region, in the beam center, scattering is most intense and then diminishes as the particle leaves the volume. The scattered light is collected by a lens system and focused onto a detector as the particle crosses the volume. The peak intensity on the detector (when the particle is in the center of the volume) is related to the size of the particle according to mie scattering theory.

Imaging type particle sizing instruments such as the OAP measure the diameter of particles by analyzing the image of a particle in the probe volume. A laser beam illuminates the particle as it crosses the instrument's probe volume. The image or shadow graph of the particle is projected through a lens system onto a light sensitive surface where the diameter of the shadow is determined. The OAP does this by counting the number of photodiodes in the shadowed region. The particle's diameter is related to the shadow diameter by a magnification factor.

counting and sizing Errors

In order for single particle counters to measure particle size distributions correctly they must be able to measure the size of particles and count them accurately. Several kinds of sizing and counting errors are examined below. These errors affect the measured size distribution differently.

Three types of sizing errors can cause shifts in the particle size distribution. First, the instrument may oversize or undersize all the particles by a constant amount. This is typically caused by shifts in the calibration. Second, the instrument may broaden the size distribution. In other words, particles of a single size may be grouped in size bins adjacent to the correct one. There are many reasons for this effect depending on the instrument. For example an uneven laser beam profile in a scattering type instrument or out of focus particles in imaging type instruments can reduce the precision of the instrument and cause broadening. The third sizing error is an instrument bias for any given size classification. For example in scattering type instruments, if the angles over which the scattered light is collected are not known precisely then larger particles will have a greater error in their measured size than smaller ones (1).

Counting errors must also be addressed because they can cause measurement bias as well. There are two varieties of counting errors. First, there is a bias toward counting (i.e. measuring) one size class of particles more than another. For example, in the OAP large particles are more likely to be seen by the instrument than smaller ones because the depth of field is 
greater for larger sizes. (This effect is corrected for in the instrument's software.) The second type of counting error occurs when particles arrive at the instrument's sample volume too rapidly to be processed. Some of the particles cross the sample volume unseen by the instrument because the instrument is busy analyzing the previous particle (dead time losses). This type of counting loss will not effect the size distribution or any of the calculated mean diameters of the particles (Sauter mean, volume mean ect.). This is because all the size classes of particles are undercounted equally. The problem occurs when number density or total mass is calculated. If there are counting losses, these measurements will be too low. Corrections for counting losses, based on instrument dead time, have been formulated and used with the FSSP (2).

The Operating Envelope

Any instrument must be operated within certain practical and theoretical bounds. These boundaries define the operating envelope of the instrument. If an instrument is yielding data with an acceptably small amount of error then the instrument is being operated within the envelope. The boundaries of the operating envelope are determined by measurement conditions, optical effects, and electronic limitations of the instrument. The latter of these two, optical effects and electronic limitations, are usually predictable and can be defined before making any measurements in the field. Measurement conditions are the most difficult aspect of the operating envelope to deal with. Measurement conditions can change during a test and cause the instrument to make sizing or counting errors without the operator's knowledge. The next section discusses how measurement conditions can effect an instrument's performance and ways of identifying when measurement conditions are outside the operating envelope of the instrument. This is followed by an explanation of the optical limitations of scattering and imaging type instruments.

\section{Measurement Conditions}

Measurement conditions that can effect the accuracy of imaging and scattering type instruments include particle size distribution, particle velocity, number density, size/velocity correlation, particle shape and refractive index, and refractive index gradients along the optical path.

Size Distribution

During a measurement of a droplet distribution within a cloud or a spray there must be a match between the range of the instrument and the distribution being measured. The instrument should not cut off the distribution. The measured distribution 
of sizes can indicate when the range of the instrument is matched to the size of the particles. It should peak toward the center of the range and tail off at the high and low end. Missing the ends of the distribution may cause errors in the calculated mean diameters. Sometimes, however, the errors are acceptable as in the case of the sauter mean diameter which is insensitive errors in counting small particles.

\section{Velocity Errors}

Particles moving at high velocities through the sample volume of scattering type instruments can be undersized. As a particle passes through the probe volume, the light it scatters is focused onto a detector. The voltage output by the detector is proportional to the light scattered by the particle as it passes through the laser beam. For example, a particle moving through a probe volume with a gaussian shaped intensity profile will scatter light and the detector measuring the scattered light will put out a voltage with a gaussian profile as the particle crosses the probe volume. A size determination is made by measuring the peak voltage. If a particle passes through the beam with a high enough velocity, the electronics within the instrument cannot respond and the signal becomes degraded (3). The signal gets spread out and the peak voltage is diminished. This results in the particle being undersized. This problem is not readily apparent by examining the data but can be avoided by knowing the velocity limitations of the instruments through laboratory testing ( 3 ).

For imaging type instruments such as the OAP a similar sort of degradation of the signal can occur (4). In this case the problem causes counting errors. This occurs because the detectors record an image that is slightly blurred. This causes the shadow to be not as dark as when the particle is moving slower. The OAP interprets this as an out of focus particle. This effectively reduces the sample volume for fast moving particles. The smallest particles are affected most. They already have a small sample volume. If their sample volume is reduced further, it can go to zero. The small particles that would fall in the first size bins never get counted. This effect can be detected by observing the smallest size bins. If counts never appear in those bins, there is a strong possibility the instrument is experiencing velocity counting errors.

It should also be noted that there is a lower limit for the velocity of particles in all single particle counters. The lower limit is several meters per second for the FSSP and OAP. This limits precludes the use of static calibration devices. 
Number Density

As with velocity, there is an upper and a lower limit for number density. Both limits can bias the data for different reasons.

The lower limit occurs when there are not enough particles present in the size bins to get a statistically valid sample. As the particles are counted and sized they are grouped together in size bins. As this process continues a particle size distribution is built up. The shape of this distribution changes from one instant to the next until enough particles populate the size bins. Any calculations yielding the mean diameter of the particles cannot be made until the general shape of the distribution stabilizes. If the number density is so small or the measurement conditions are changing so rapidly that the distribution does not stabilize during the duration of the experiment, then the data will be questionable.

Extremely high number density can effect the data from scattering and imaging type instruments. For both instruments, high number density causes counting losses and coincidence errors. Additionally, scattering type instruments can be affected by laser beam attenuation.

High number density can cause counting losses in both types of instruments. After a particle passes through the sample volume, the instrument must make a size determination. During this time (the so called dead time) the instrument is insensitive to particles crossing the sample volume and some of the particles are not counted. As previously mentioned this has no effect on the distribution but it does effect number density and total mass measurements and some corrections algorithms have been developed (2).

Even moderate number densities can cause counting losses if the velocity of the particles is high. One possible way to determine when high number density is causing counting losses is to measure the instruments dead time while data is being gathered as is done in the FSSP (5). If the ratio of dead time to measurement time approaches unity then counting losses are significant.

Coincidence is both a sizing and a counting error that occurs when more than one particle is in the instrument's sample volume at a time. As number density increases the probability of coincidence errors increases. Most scattering type instruments cannot detect coincidence errors. The instrument will measure two smaller particles as one large one. This effect tends to skew the size distribution towards larger sizes. This error is difficult to detect. One method is to measure (or estimate) the 
total mass using an independent method and use the measured distribution to calculate the average number density. The reciprocal of number density gives the average volume occupied by each particle. If this volume is close to size of the probe volume then there is a high probability coincidence errors are occurring.

Laser beam attenuation occurs when there are so many particles in the laser beam (not necessarily in the sample volume) that the intensity of the beam is attenuated. This diminishes the intensity of the light on the particles in the sample volume. These particles will scatter less light and therefore they will be undersized by scattering type instruments. Laser beam attenuation is not a problem with imaging type instruments.

Size Velocity correlation

A size velocity correlation occurs when particles in any given size bin are moving at a velocity that is different than those in the other size bins. In any single particle counter, a size velocity correlation causes data to be biased towards faster moving particles.

The reason for this bias is faster moving particles have a greater probability of crossing the sample volume than slower moving ones (6). This bias is more a property of the measurement process (called a temporal or flux sensitive measurement) than an instrument error. However, data from single particle counters must be corrected before it can be compared with other types of instruments that are not sensitive to the particles' velocity (7). Also, measurements should be corrected before calculating number density or total mass.

For the FSSP the size velocity correlation creates one additional problem. The FSSP must have all particles traveling at the same velocity in order to correctly define its sample volume. One dimension of the sample volume in the FSSP is determined by the average transit time of the particles through the laser beam. Particles with transit times shorter than the average are rejected because they may have an edge effect error. The FSSP will not only reject these particles but it will also reject particles that are moving rapidly. Both will have transit times that are shorter than the average. This effectively reduces the sample volume for fast moving particles. Any measurements with the FSSP in environments with a size velocity correlation should be avoided. 
Particle Shape and Index of Refraction

Shape and index of refraction are two important factors that determine a particle's light scattering pattern. Scattering instruments will preform poorly if particles are irregularly shaped or have an unknown index of refraction.

one situation where irregular shaped particles are encountered is in supercooled clouds where ice crystals are present. The FSSP performs very poorly if any ice is present. The particle size distributions are often flat and populate all the size bins. It is theorized that the ice crystals act as tiny prisms and reflectors. Each crystal sends reflected and refracted rays of light in random directions depending on their orientation. Some of these rays enter the collecting optics of the FSSP and are interpreted as particles. Thus one ice crystal may cause counts in many different size bins depending on its orientation in the beam.

Other situations where irregularly shaped particles may be encountered is with glass beads. They can be broken, chipped or have air bubbles in them. This again could lead to counting and sizing errors in scattering type instruments.

Spherical particles whose index of refraction is unknown will cause sizing errors but not counting errors. The sizing errors depend on the difference between the expected index of refraction and the actual one. A distribution of particles with an unknown index of refraction can be undersized, oversized, or measured correctly depending on the size range in question.

Irregularly shaped particles or particles whose index of refraction is unknown generally do not pose a severe problem for imaging type instruments. Although it is difficult to classify irregularly shaped particles with any one number, such as diameter, they do not cause the sizing and counting errors that are seen in scattering type instruments.

Refractive Index Gradients

Refractive index gradients along the optical path will deviate the laser beam away from a straight line. This depends on the severity of the gradient and the total distance the beam must travel in the gradient. This beam steering effect can cause temporary changes in the optical collection angles similar to those caused by misaligning the laser beam. 


\section{optical Effects}

optical effects determine the operating envelope of the instrument by placing fundamental restrictions on accuracy and precision of the measured size of the particle. Scattering type instruments are restricted by such factors as a multivalued Mie response function, large particle errors, edge effect errors, and laser beam fluctuations. Imaging instruments are limited by Fresnel diffraction and depth of field errors.

Mie Response Function

A plot of the Mie response function for the FSSP is shown in figure 1. There are several features to note about this figure that are common to all scattering type instruments. In the region where the diameter of the particle is smaller than the wavelength of light the scattering pattern increases as diameter to the sixth power. This is known as the Rayleigh region of the curve. If the diameter of the particles is on the order of the wavelength of the light, this is known as the Mie region. In this region there is not a definite relationship between the diameter of the particle and the intensity of the scattered light. Particles much larger than the wavelength have a scattered intensity that goes as the diameter squared. This region of the curve can be calculated by geometrical optics and Fraunhofer diffraction theory.

Particles falling in the Rayleigh region are difficult for scattering instruments to measure because of the large dynamic range needed for the detectors. For example, to cover the size range from .1 to 1 micrometer would require a detector with a dynamic range of a million to one. Also, particles in this range scatter so little light that the laser power must be quite high to get an adequate signal. If the particles absorb this energy they will quickly vaporize.

Particles falling in the Mie region are also difficult to size using scattering techniques. In this region the Mie response function is multivalued. Particles of different sizes can scatter the same amount of light. This places fundamental limitations on the ability to resolve different diameter particles. Several techniques can be used to help alleviate this problem. One way is to group the particles that give the multivalued response in the same size bin. Another way is to use a multimodal laser beam to wash out the bumps in the Mie region as the FSSP does (8). A third way is to use a multiple frequency laser, this will again wash out the bumps in the Mie curve. 


\section{Large Particle Errors}

When Mie theory is used to calculate the scattered intensity from a particle, one of the assumptions is the light illuminating the particle is a plane wave of uniform intensity. This assumption is valid if the particle is much smaller than the focused laser beam. For particles that are about the same size or larger than the diameter of the laser beam, additional corrections to Mie theory are necessary (9).

The scattering pattern from large particles in a gaussian beam is quite different from small ones. Figure 2 shows the scattered intensity collected over a solid angle. This plot was made by moving an aperture from a dark field reticle (10) through the cross section of a focused gaussian laser beam. At each location the aperture scattered light in a manner similar to that of a spherical absorbing particle. The light was collected over a solid angle and the intensity was plotted as a function of position within the beam. The pattern is unusual but can be predicted with Fraunhofer diffraction theory if the gaussian intensity profile of the beam is also modeled in the calculation.

The implication of this is, particles that are large and outside of the range of the instrument could be mistaken as two smaller size particles. If a large particle traverses the center chord of the laser beam, it will produce a signal with two peaks. This could cause a scattering type instrument to interpret a large particle as two smaller ones, thus producing a sizing and a counting error.

\section{Edge Effect Errors}

Edge effect errors occur when a particle crosses a chord of the laser beam close to the outside edge where the intensity of the beam is less. This causes undersizing of the particle in scattering type instruments. As mentioned previously the FSSP solves this problem by determining the transit time of the particle in the beam. Particles crossing the edge have a shorter transit time and are rejected. Another method to limit edge effect errors makes use of a pointing beam to define a region of near constant intensity in a larger beam (11). Particles are only measured if they go through the pointing beam which is in the center of a much larger gaussian shaped beam.

Both methods still cause sizing errors if there are intensity variations across the sample volume (12). This will cause broadening of the size spectrum.

A third method to eliminate edge effect errors makes use of intensity deconvolution (13). This method assumes that each size class of particles will traverse all possible chords of the laser beam. Using this, the size distribution can be calculated from 
the scattered intensity. Although this method requires more data processing and detailed measurements of the sample volume, it does not suffer from the same problems encountered by either of the other two methods.

Laser Fluctuations

since scattering type instruments measure the intensity of the scattered light, sizing errors can occur if anything changes the intensity profile. The most common are dirty optical components, loss of laser power over time, short term laser fluctuations and misaligned optical components. In general these effects tend to cause undersizing of the particles.

In the FSSP correction for laser power fluctuations is achieved by monitoring the power within cavity of the laser with a photodiode. Corrections to the measured size of the particle are made on line.

\section{Fresnel Diffraction}

Fresnel diffraction imposes an accuracy limit on imaging type instruments. These instruments must use some criteria such as a shadow boundary to define the edge of a particle in order to make a size determination. Fresnel diffraction blurs this boundary and makes it impossible to determine the exact size. The wavelength of the light illuminating the particle is the theoretical limit.

Depth of Field

The depth of field in imaging type instruments goes as the square of the diameter of the particle. This means that large particles remain in focus over a greater distance and can be sized more accurately than small ones. The sample volume of many imaging type instruments such as the OAP is a function of the diameter of the particle. When the instrument tabulates the particle size distribution there must be a correction for the varying sample volume. Imaging type instruments are better suited for measuring larger particles than smaller ones. 


\section{Conclusion}

To ensure accurate measurement of particle size distributions with scattering and imaging type instruments, the operating envelope must not be exceeded. In order to stay within the operating envelope the user must first know which variables can cause errors, identify how these variables can effect the instrument, and finally recognize when any measurement condition has affected the data. If the theoretical and practical limitations of these instruments are not exceeded, they can make accurate, nonintrusive measurements of particle size distributions in a flow.

\section{REFERENCES}

1. Pinnick, R.G., D.M. Garvey and L.D. Duncan (September 1981). Calibration of Knollenberg FSSP Light-Scattering Counters for Measurement of Cloud Droplets. J Appl Meteor. 20: 1049-1057.

2. Baumgardner, D., W. Strapp and J.E. Dye (1985). Corrections for the Forward scattering spectrometer probe. part II: Corrections for Coincidence and Dead-Time Losses. J Atmos and Oceanic Tech. 2: 626-632.

3. Cerni, T.A. (1983). Determination of the size and Concentration of Cloud Drops with an FSSP. J Climate Appl Meteor. 22: 1346-1355.

4. Baumgardner, D. (1987). Corrections for the Response Times of Particle Measuring probes. Proc. 6th Symposium Meteor. Obs. and Instr., New Orleans, La: 148-151.

5. Forward Scattering spectrometer Probe, PMS Model FSSP-100, operating and Servicing Manual. Particle Measuring systems, Inc., Boulder, Co, 1984 .

6. Young, B.W., and W.D. Bachalo (1987). The Direct Comparison of Three In-Flight Droplet sizing Techniques for Pesticide spray Research. International symposium on optical Particle sizing: Theory and practice, Rouen, France.

7. Bachalo, W.D., M.J. Houser, and J.N. Smith (1986). Evolutionary Behavior of Sprays Produced by Pressure Atomizers. AIAA 24 th Aerospace Science Meeting. Reno, Nevada: paper 86.0296.

8. Knollenberg, R.G. (1976). Three New Instruments for Cloud Physics Measurements. Preprints Int. Conf. Cloud Physics, Amer. Meteor. Soc., Boulder, CO: 554-561.

9. Grehan, G., B. Meyhew., and G. Gouesbet (1986). Localized Approximation to the Generalized Lorenz-Mie Theory and its Application to Optical Particle Sizing. ICALEO 1986 proceedings. 58: 189-196. 
10. Hovenac, E. A. (Nov. 1986). Use of Rotating Reticles for Calibration of Single Particle counters. ICALEO 1986 Proceedings. 58: 129-134.

11. Hess C.F. (1984). Nonintrusive Optical Single Particle Counter for Measuring the size and Velocity of Droplets in a spray. Applied optics. Vol 23, No.23: 4375-4382.

12. Baumgardner, D. (1987). Droplet sizing and counting Limitations of PMS Probes. ILASS 87 ist Annual Conference on Liquid Atomization and Spray Systems. Madison, WI.

13. Holve, D.J. and K.D. Annen (1984). Optical Particle counting, Sizing and Velocimetry Using Intensity Deconvolution. Optical Engineering. Vol 23, No. 5: 591-603. 


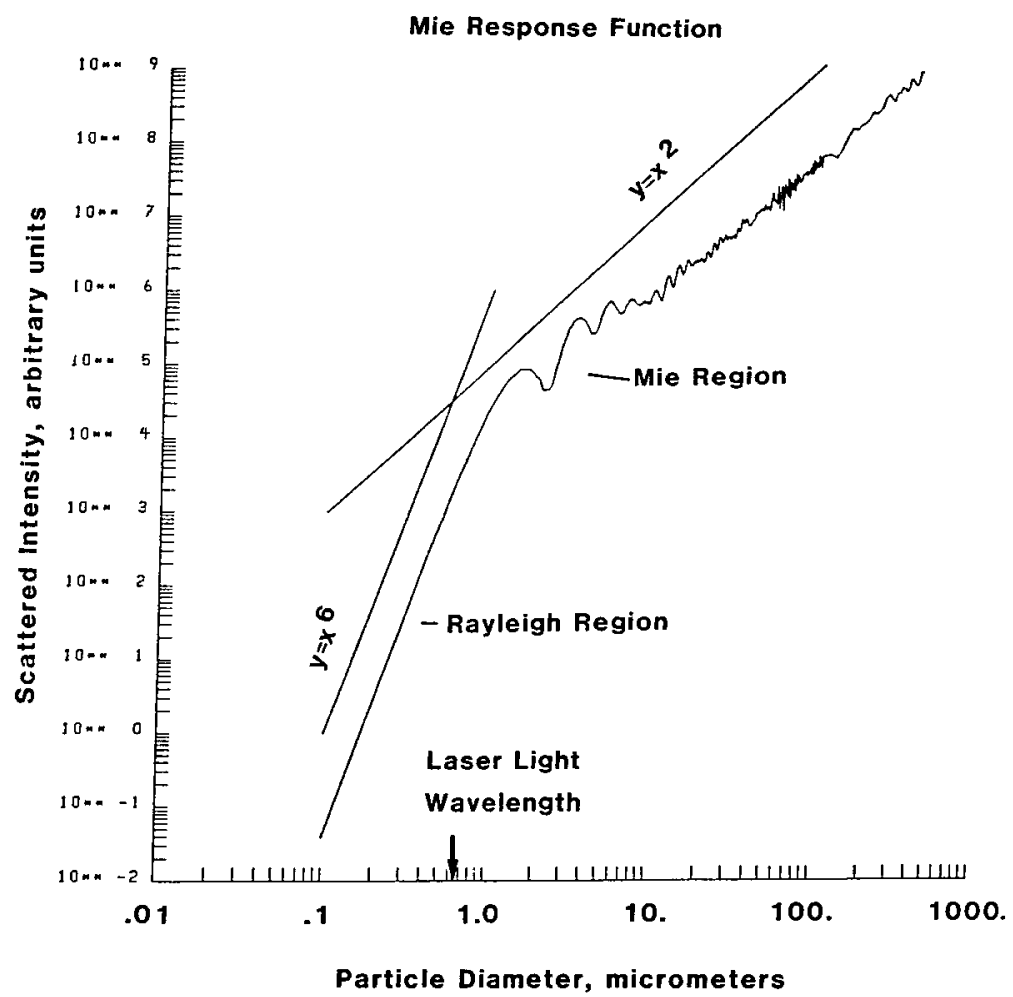

FIGURE 1. - MIE RESPONSE FUNCTION FOR A SCATTERING TYPE INSTRUMENT LIKE THE FSSP.

Scattered Light Intensity as a Function of Aperture Position

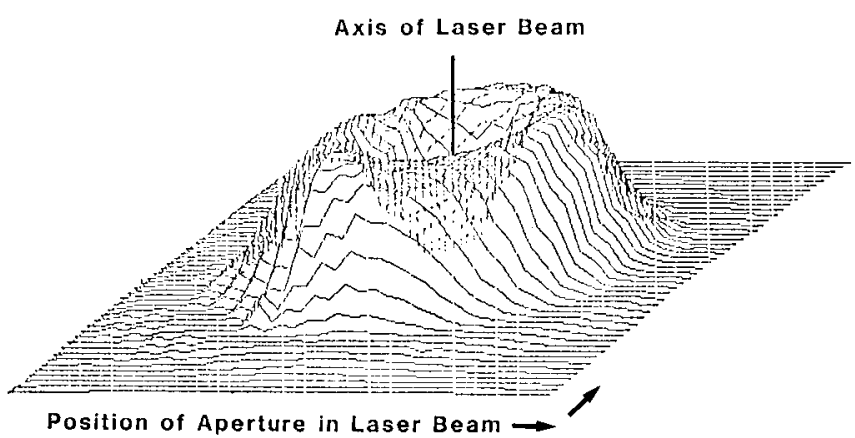

FIGURE 2. - SCATTERED LIGHT COLLECTED OVER A RANGE OF ANGLES AS A FUNCTION OF THE POSITION OF AN APERTURE WITHIN THE LASER BEAM. 


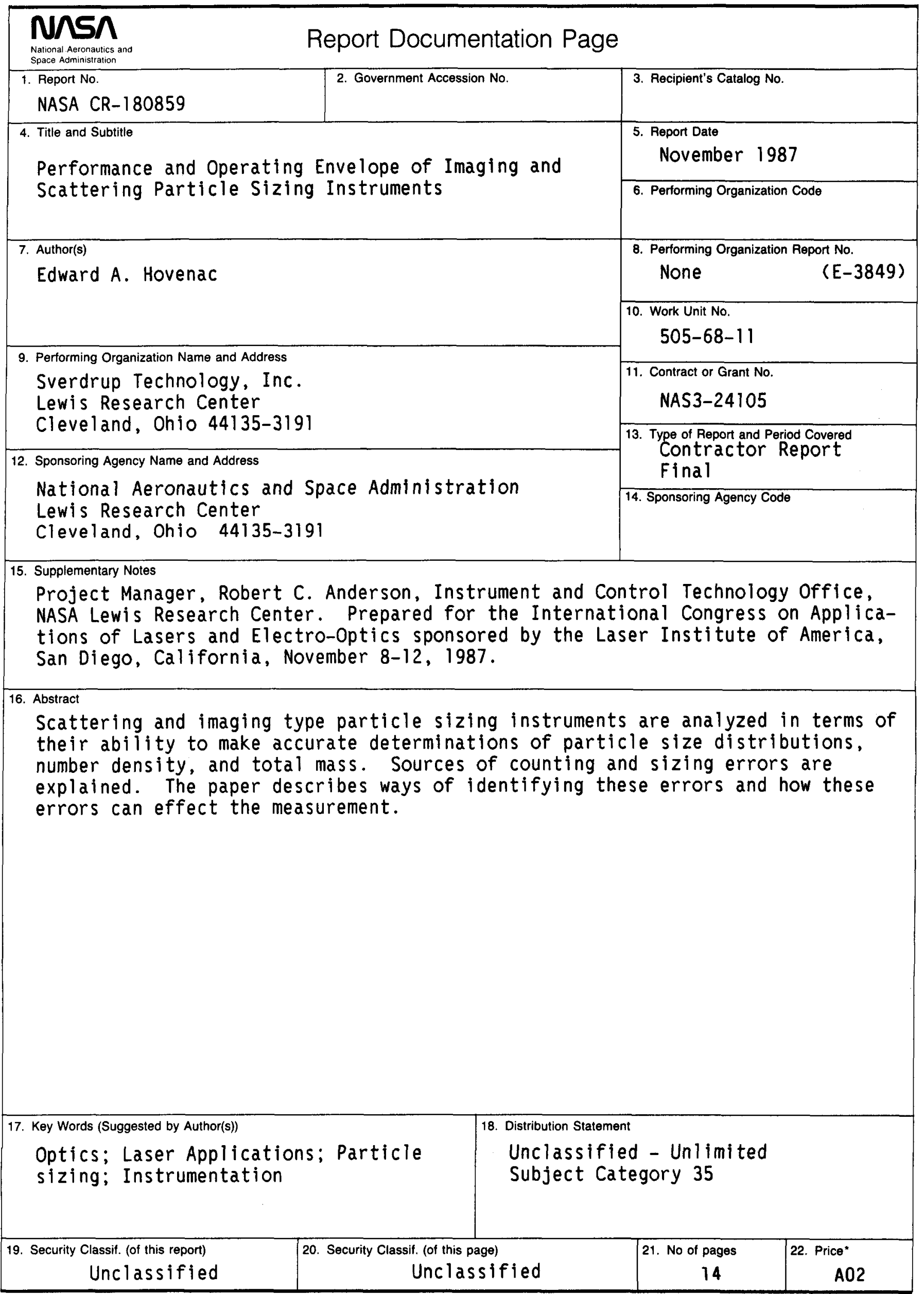

